\title{
Beyond the notes
}

\author{
The way performers shape notes brings music to life. \\ Nicholas Cook argues that measuring these subtle changes \\ can help us appreciate and replicate the performer's art.
}

T he art of musical performance lies largely in nuance - in making notes longer or shorter than they are written, or in shaping their dynamics, articulation or pitch. Performers don't generally have explicit theories of these things, as it's all done by ear. But these often subtle changes to the written score are responsible for a great deal of what makes music memorable, moving and meaningful - and they can be measured.

This combination of cultural meaning and measurability makes musical performance a productive example of the relationship between science and the humanities. When musicology came into being in the nineteenth century, it was modelled on philology, the study of ancient texts. For this reason, musicologists have tended to think of music as a form of writing. But much of what performers do, and what listeners respond to, falls between the notes as musicologists construe them. This is where science comes in.

Measurements cannot capture cultural values, but people listening to music respond to specific sounds. These sounds are amenable to scientific study, providing insights into the cultural values they embody. I focus on classical piano performance, but my claim is more general: to understand music as performance, we must use scientific and humanities approaches in tandem.

\section{Stick to the plot}

Two of the most important aspects of musical performance are the shaping of the tempo and the dynamics. Tempo shaping is the lengthening or shortening of notes or phrases and is measured by extracting beat durations from sound. Dynamics shaping is the patterning of loud and soft notes, to create one-off accents or waves of increase and decrease. It can be extracted as a continuously varying value or as a series of discrete values associated with individual notes.

Musicologists and psychologists have generally focused on how such data relate to the structure of music, drawing on traditional, notation-based analytical methods. For instance, reading by means of a kind of reverse engineering from the performance back to the composition, they have shown how performers use various combinations of speed change and dynamic accents to underline structural breaks or bring out important points.

Line graphs of tempo and dynamics are hard to relate to the music we hear, but over the past two years software has been developed that incorporates these graphs within a music visualization program so that they scroll past a cursor as one listens. Other limitations to this type of approach are less tractable. If performance is analysed in terms of the score-based structure, one is deaf to aspects of the performance that have nothing to do with what is written down. In effect, this assumes that the point of performance is to reproduce a meaning that is already there on the printed page, but any jazz or pop performance demonstrates what an inadequate approach that is.

There is a further, more subtle, problem. Try dancing to a Chopin mazurka and it soon becomes clear that concert evocations of dance music have much more extravagant shaping than music that is for actual dancing. A tempo graph would show this, and so says something about the music one experiences. Its shape, however, is the result of several distinct factors. To understand what is going on, we need to break the data down into their component parts. The question is what those parts might be.

\section{Musical movement}

In the early 1990s, Henkjan Honing and Peter Desain suggested that the shaping of both tempo and dynamics in classical music performance can be explained in terms of three main components: note-to-note shaping, the composer's 'pulse', and hierarchical phrase arching. The third of these refers to the way performers get faster and louder as they play into a phrase, and softer and quieter as they come out of it, giving the music a kind of breathing quality. It is often seen in nineteenth-century piano music, such as Chopin's. It is hierarchical in that such patterns can be found at multiple levels — such as 2, 4, 8 and even 16 bars. It is widely seen as part of what it means to play 'musically', that is to say expressively and meaningfully.

Musicologists tend to be suspicious of such generalizations. What is considered 'musical' has varied throughout history, as have practices of performance. My team at the AHRC 
